\title{
Cinética de crecimiento in vitro de dos cepas de Trypanosoma cruzi (Kinetoplastida: Trypanosomatidae) en células miocárdicas de roedores con diferente susceptibilidad
}

\author{
Rafael-Oswaldo Angel B. ${ }^{1}$, Misael Chinchilla C. ${ }^{2 *}$, Olga Marta Guerrero B. ${ }^{2}$ \\ \& Alfredo Castro C. ${ }^{2}$ \\ 1 Departamento de Microbiología, Facultad de Medicina, Universidad de El Salvador. Fax: (503) 225-8822; rangel@ \\ elsalvador.com \\ 2 Centro de Investigación en Enfermedades Tropicales, CIET, Departamento de Parasitología, Facultad de \\ Microbiología, Universidad de Costa Rica, 2060, San José, Costa Rica. Teléfono 506-207-42-77. Fax: 506-225-23- \\ 74. *Correspondencia.
}

\author{
Recibido 22-VII-2004. Corregido 18-XI-2005. Aceptado 11-V-2006.
}

\begin{abstract}
In vitro growth kinetics of two Trypanosoma cruzi (Kinetoplastida: Trypanosomatidae) clones in myocardial cells from rodents of different susceptibility. Two Trypanosoma cruzi isolates, TCR-4 from Costa Rica and UES-1 from El Salvador, were studied in vitro to compare their infectivity or resistance and intracellular replication in myocardial cells in three strains of mice and rats: NGP white mice, $\mathrm{C}_{3} \mathrm{H}$ mice and Sprague Dowley rats. Myocardial cells were cultured on coverslips at $37^{\circ} \mathrm{C}$ in a humid $10 \% \mathrm{CO}_{2}$ atmosphere and then infected at a ratio of one tripomastigote per cell. Samples were studied after 24, 72, 96 and $120 \mathrm{~h}$ of infection to determine parasite infection capacity and intracellular multiplication. Both parasites had the highest infection capacity in $\mathrm{C}_{3} \mathrm{H}$ mice, followed by NGP mice cells with a very low infection rate. Lastly, almost no Trypanosoma cruzi multiplication was observed in Sprague Dowley rats, suggesting a strong natural resistance in this animal to both strains of the parasite. The UES-1 isolate presented higher multiplication and greater invasion than the TCR-4 strain, showing greater virulence of UES-1 in heart cells, at least in vitro. Rev. Biol. Trop. 55 (1): 121-126. Epub 2007 March. 31.
\end{abstract}

Key words: Trypanosomatidae, Trypanosoma cruzi, heart cells, cell culture.

Durante su complejo ciclo de vida, Trypanosoma cruzi alterna entre hospederos invertebrados y vertebrados en los cuales establece la infección (Zingales y Colli 1985). Las formas encontradas en los vertebrados son parásitos intracelulares obligados que invaden y se replican en una gran variedad de células fagocíticas y no fagocíticas (Sadigursky 1999, Scharfstein y Morrot 1999). Aunque T. cruzi puede parasitar cualquier tipo de célula, su desarrollo en las células musculares cardiacas es importante, ya que su presencia en este tipo de células tiene un papel preponderante en las manifestaciones clínicas de la enfermedad de Chagas (Andrade 1999b). También se ha determinado que existe una participación muy activa de las células cardiacas en su respuesta a la infección con T. cruzi, ya que al cultivarlas, en presencia de varias citoquinas, se observan diferencias importantes en la multiplicación del parásito en ellas (Postani et al. 1999). También es importante mencionar que las células de diferentes animales o aún de diferentes órganos en el mismo animal, presentan variaciones en el crecimiento de T. cruzi, lo cual ha sido demostrado en ciertas especies de roedores (Araujo et al. 1976, Chinchilla et al. 1995). Sobre la base de estos estudios y empleando un modelo in vitro descrito por Reyes y Chinchilla (1987), este estudio compara la multiplicación de dos 
cepas de $T$. cruzi en células miocárdicas de varias especies de roedores.

\section{MATERIALES Y MÉTODOS}

Parásitos: Se utilizaron dos cepas, un aislamiento de T. cruzi de El Salvador (UES-1) obtenido de un niño en fase aguda en el departamento de Santa Ana, El Salvador en 1999 y otro aislamiento hecho en Costa Rica en 1987 (TCR-4) de un ejemplar de Triatoma dimidiata; ambas cepas son mantenidas por pases sucesivos cada 25 días en ratones $\mathrm{C}_{3} \mathrm{H}$.

Animales: Se utilizaron ratones $\mathrm{C}_{3} \mathrm{H}$ de 20-25 g de peso para la obtención de tripomastigotos. Además ratones blancos NGP, ratones $\mathrm{C}_{3} \mathrm{H}$ y ratas Sprague Dowley de 2-4 días de nacidos para los correspondientes cultivos de células.

Cultivo de células miocárdicas y modelo de infección: Se realizó una disección de los animales recién nacidos bajo condiciones asépticas y los corazones se colocaron en una solución de Hanks (HBSS) a $4^{\circ} \mathrm{C}$, lavándose una vez en dicha solución y para posteriormente cortarlos en pequeñas porciones y luego colocarlos cuidadosamente en frascos de Erlenmeyer para su tripsinización por $10 \mathrm{~min}$ a $37{ }^{\circ} \mathrm{C}$. Este procedimiento se repitió dos veces más y se recolectó al final del proceso el sobrenadante en Minimal Essential Medium (SIGMA ${ }^{\circledR}$ ) más $10 \%$ de suero fetal bovino con 10-100 UI de penicilina, $0.01 \mathrm{mg}$ de estreptomicina y 0.025 $\mu \mathrm{g}$ de anfotericina B por ml (MEM-SFB). La suspensión celular se centrifugó a 800 g por 10 min, se descartó el sobrenadante y el sedimento celular se resuspendió en $10 \mathrm{ml}$ de MEMSFB, colocándose posteriormente en botellas de cultivo estériles, las cuales se incubaron a $37^{\circ} \mathrm{C}$ con una atmósfera de $5 \%$ de $\mathrm{CO}_{2}$ por 24 h. Luego se agregaron $8 \mathrm{ml}$ de MEM-SFB, se volvió a incubar por otras $24 \mathrm{~h}$ y después de este tiempo, se descartó el medio líquido, se agregaron $10 \mathrm{ml}$ de medio y se incubó por 24 a $48 \mathrm{~h}$ hasta que se formara una monocapa. Para pasar las células a cubreobjetos, las botellas de cultivo se trataron con tripsina $0.25 \%$ a $37{ }^{\circ} \mathrm{C}$ con una atmósfera de $5 \%$ de $\mathrm{CO}_{2}$ hasta que la monocapa se desprendiera. Posteriormente se colocó $0.1 \mathrm{ml}$ de esta suspensión en cubreobjetos 22 × $22 \mathrm{~mm}$ y se incubaron $24 \mathrm{~h}$ a $37^{\circ} \mathrm{C}$ con una atmósfera de $5 \%$ de $\mathrm{CO}_{2}$ para su infección posterior (Reyes y Chinchilla 1987). Después de incubar por $24 \mathrm{~h}$ las células en cubreobjetos se infectaron con $0.1 \mathrm{ml}$ de suspensión de tripomastigotos sanguíneos (derivados de ratones $\mathrm{C}_{3} \mathrm{H}$ infectados previamente con las respectivas cepas) en MEM-SFB, en una proporción de un parásito por célula miocárdica y se incubaron a $37{ }^{\circ} \mathrm{C}$ con 5-10\% de una atmósfera de $\mathrm{CO}_{2}$. Los cubreobjetos se retiraron (por duplicado) a las 24, 72 y 96 h lavándolos cuidadosamente con HBSS y fueron teñidos adecuadamente con Giemsa ( $1 \mathrm{ml}$ de colorante de Giemsa más 9 partes de buffer de fosfatos pH 7.2). Los controles consistieron en cultivos de células miocárdicas de animales sanos de cada especie de roedor. Cada cubreobjeto se examinó por microscopía de luz (lente $100 \mathrm{x}$ ) contándose en cada uno 500 células; de esta manera se estableció el número de células infectadas y el número de parásitos intracelulares totales lo que permitió calcular el número de parásitos por 100 células (población total), el número de parásitos por células infectadas y la tasa de multiplicación. Este valor se calculó dividiendo el número de parásitos intracelulares después de $120 \mathrm{~h}$ entre el número de parásitos después de $24 \mathrm{~h}$. Las diferencias observadas entre los índices de reproducción del parásito en las células de los tres animales se evaluaron mediante la prueba de $t$ de Student para comparación de promedios independientes utilizando niveles de confianza del $95 \%$.

\section{RESULTADOS}

En los cuadros 1 y 2 se observa la multiplicación de los aislamientos de $T$. cruzi en células de corazón de los tres roedores, ratón $\mathrm{C}_{3} \mathrm{H}$ $\left(\mathrm{RT} \mathrm{C}_{3} \mathrm{H}\right)$, ratón NGP (RT NGP) y rata blanca Sprague Dowley(R). En los tres animales la cantidad de parásitos por células cardiacas infectadas a las $24 \mathrm{~h}$ fue similar, pero el número 
CUADRO 1

Curva de crecimiento de T. cruzi TCR-4 en células cardiacas

TABLE 1

Growth curve of T. cruzi TCR-4 in heart cells

\begin{tabular}{|c|c|c|c|c|c|c|}
\hline \multirow{2}{*}{ Animal } & \multirow{2}{*}{$\begin{array}{l}\text { Tiempo de } \\
\text { infección }\end{array}$} & \multicolumn{2}{|c|}{ Células contadas } & \multicolumn{3}{|c|}{ Parásitos } \\
\hline & & Totales & Infectadas & Intracelulares & /100 células & /100 células infectadas \\
\hline \multirow{4}{*}{$\mathrm{R}$} & 24 & 501 & 2 & 2 & 0.4 & 100 \\
\hline & 72 & 503 & 1 & 1 & 0.2 & 100 \\
\hline & 96 & 500 & 4 & 4 & 0.8 & 100 \\
\hline & 120 & 500 & 4 & 5 & 1.0 & 125 \\
\hline \multirow{4}{*}{ RT NGP } & 24 & 510 & 2 & 3 & 0.6 & 150 \\
\hline & 72 & 500 & 4 & 6 & 1.2 & 150 \\
\hline & 96 & 505 & 8 & 16 & 3.2 & 200 \\
\hline & 120 & 503 & 10 & 28 & 5.6 & 280 \\
\hline \multirow{4}{*}{ RT $\mathrm{C}_{3} \mathrm{H}$} & 24 & 504 & 15 & 15 & 3.0 & 100 \\
\hline & 72 & 507 & 19 & 49 & 9.7 & 258 \\
\hline & 96 & 515 & 25 & 75 & 14.6 & 300 \\
\hline & 120 & 510 & 56 & 425 & 83.3 & 759 \\
\hline
\end{tabular}

RT $\mathrm{C}_{3} \mathrm{H}$ : ratón $\mathrm{C}_{3} \mathrm{H}$; RT NGP: ratón NGP; R: rata.

RT $\mathrm{C}_{3} \mathrm{H}: \mathrm{C}_{3} \mathrm{H}$ mouse; RT NGP: NGP mouse; R: rat.

TCR-4-cepa de Costa Rica.

TCR-4-parasite clone from Costa Rica.

de parásitos intracelulares a las 72, 96 y 120 fue diferente y mucho más obvio en las células cardiacas de $\mathrm{RT} \mathrm{C}_{3} \mathrm{H}$ a las $72 \mathrm{~h}$ después de la infección, especialmente las células infectadas con la cepa UES. En las células de corazón de $\mathrm{R}$, el número de parásitos intracelulares permaneció casi constante durante el experimento, aunque como se observa en el cuadro 2 la multiplicación de esta cepa (UES) fue muy baja. Es necesario señalar que después de 120 $\mathrm{h}$, la mayoría de las formas intracelulares fueron amastigotos; sin embargo, en pocos casos, se observaron tripomastigotos en el interior de las células, especialmente en lo referente a las células cardiacas de $\mathrm{RT}_{3} \mathrm{H}$, en las que se observaron tripomastigotos libres como resultado de la alta multiplicación del parásito y probable lisis de algunas células. En el cuadro 2 se observa que la cepa TCR-4 infectó menor cantidad de células en comparación con la cepa UES. Un comportamiento similar se observó con respecto a la multiplicación intracelular de la cepa TCR-4.

La tasa de multiplicación de los parásitos por 100 células cardiacas infectadas indican una mayor multiplicación de la cepa $T$. cruzi (UES) en $\mathrm{RT}_{3} \mathrm{H}$ que la cepa TCR4. Aunque en las células de corazón de $\mathrm{R}$ se evidenció multiplicación, ésta fue muy baja para ambas cepas (Cuadro 3). La tasa de multiplicación 24/120 h en células de corazón de RT $\mathrm{C} 3 \mathrm{H}$ fue mayor para $T$. cruzi (UES) que el mostrado por la otra cepa (83.3) (Cuadro 3). Esta misma situación se repite también a las 72 y 96 h de infección, no obstante valores de multiplicación intermedios fueron observados en células de corazón de RT NGP. Puede verse también que hubo una mayor multiplicación en las células de corazón de RT NGP por parte de T. cruzi (UES) que la mostrada por T. cruzi (TCR-4); ambas cepas mostraron índices de multiplicación similares en células de corazón de $\mathrm{R}$ (Cuadros 1 y 2). 
CUADRO 2

Curva de crecimiento de T. cruzi UES en células cardiacas

TABLE 2

Growth curve of T. cruzi UES in heart cells

\begin{tabular}{|c|c|c|c|c|c|c|}
\hline \multirow{2}{*}{ Animal } & \multirow{2}{*}{$\begin{array}{l}\text { Tiempo de } \\
\text { infección }\end{array}$} & \multicolumn{2}{|c|}{ Células contadas } & \multicolumn{3}{|c|}{ Parásitos } \\
\hline & & Totales & Infectadas & Intracelulares & /100 células & /100 células infectadas \\
\hline \multirow{4}{*}{$\mathrm{R}$} & 24 & 505 & 2 & 2 & 0.4 & 100 \\
\hline & 72 & 503 & 2 & 2 & 0.4 & 100 \\
\hline & 96 & 500 & 4 & 4 & 0.8 & 100 \\
\hline & 120 & 510 & 4 & 5 & 1.0 & 125.0 \\
\hline \multirow{4}{*}{ RT NGP } & 24 & 500 & 4 & 5 & 1.0 & 125 \\
\hline & 72 & 500 & 14 & 18 & 3.6 & 129 \\
\hline & 96 & 500 & 12 & 48 & 9.6 & 400 \\
\hline & 120 & 500 & 32 & 126 & 25.2 & 394 \\
\hline \multirow{4}{*}{$\mathrm{RT} \mathrm{C}_{3} \mathrm{H}$} & 24 & 500 & 16 & 22 & 4.4 & 138 \\
\hline & 72 & 500 & 39 & 78 & 15.6 & 200 \\
\hline & 96 & 500 & 51 & 215 & 43.0 & 422 \\
\hline & 120 & 500 & 62 & 892 & 178.4 & 1439 \\
\hline
\end{tabular}

RT $\mathrm{C}_{3} \mathrm{H}$ : ratón $\mathrm{C}_{3} \mathrm{H}$; RT NGP: ratón NGP; R: rata.

RT $\mathrm{C}_{3} \mathrm{H}: \mathrm{C}_{3} \mathrm{H}$ mouse; RT NGP: NGP mouse; R: rat.

UES. cepa de El Salvador.

UES. parasite clone from Costa Rica.

CUADRO 3

Multiplicación de cepas de T. cruzi en células cardiacas

TABLE 3

Multiplication of $\mathrm{T}$. cruzi parasite clones in heart cells

\begin{tabular}{cccc} 
T. cruzi & Animal & \multicolumn{2}{c}{ Parásitos/100 células (Infección con T. cruzi) } \\
& RT C ${ }_{3} \mathrm{H}$ & $120 \mathrm{~h}$ & Tasa120/24 h \\
UES* & 4.4 & 178.4 & $\mathbf{4 0 . 5}$ \\
RT NGP & 1 & 25.2 & $\mathbf{2 5}$ \\
R & 0.4 & 1 & $\mathbf{1 . 2 5}$ \\
RT C ${ }_{3} \mathrm{H}$ & 3 & 83.3 & $\mathbf{2 7}$ \\
RT NGP & 1 & & $\mathbf{9 . 3 3}$ \\
R & & 25.0 & $\mathbf{1}$
\end{tabular}

RT $\mathrm{C}_{3} \mathrm{H}$ : ratón $\mathrm{C}_{3} \mathrm{H}$; RT NGP: ratón NGP; R: rata.

$\mathrm{RT}_{3} \mathrm{H}$ : $\mathrm{C} 3 \mathrm{H}$ mouse; RT NGP: NGP mouse; R: rat.

* cepa del El Salvador

* parasite clone from El Salvador

** cepa de Costa Rica

** parasite clone from Costa Rica 


\section{DISCUSIÓN}

Se ha propuesto que la diversidad molecular existente en cepas de T. cruzi puede afectar el funcionamiento de los tejidos y órganos que infectan, como se evidencia por el hecho de que infecciones con cepas de parásitos pertenecientes a diferentes grupos fenotípicos, inducen distintos patrones histopatológicos en ratones infectados (Andrade 1999a, De Luca et al. 1993). Es así como estudios desarrollados con cepas de laboratorio sugieren que la expresión variable de la enfermedad de Chagas puede ser influenciada por la genética y la diversidad biológica de clones de T. cruzi, circulantes en reservorios selváticos y domésticos (Macedo y Pena 1998, Magalhaes et al. 1996). La posibilidad de variantes en la virulencia debida a la presencia de clones claramente definidos se ha comprobado, por lo menos en la cepa salvadoreña, ya que se han determinado clones con diferente grado de infectividad para macrófagos (Calderón-Arguedas et al. 2002). En Centroamérica no se han llevado a cabo estudios de este tipo, debido probablemente a que los cuadros clínicos descritos en algunos países no son tan dramáticos como los ocurridos en Sudamérica, aunado a la falta de recursos económicos en los institutos de investigación (Bloch 1982).

El patrón de crecimiento típico en células cardiacas de ratones $\mathrm{C}_{3} \mathrm{H}$ por parte de $T$. cruzi fue descrito en los trabajos realizados por Reyes y Chinchilla (1987). Estos investigadores utilizaron solamente una especie de animal, pues los objetivos eran otros, mientras que en este estudio se compara tal multiplicación en diferentes cepas de animales de laboratorio. La diferencia en multiplicación de $T$. cruzi en los tres tipos de animales (Cuadro 3), nos permite razonar lo siguiente: debido a que las células cardiacas no realizan mecanismos de defensa como los macrófagos (Bogdan y Rollinghof 1999, Dos-Reis 1997, Israelski et al. 1990), los resultados indican una resistencia natural a la infección de las células cardiacas de ratón en NGP y más marcada en rata blanca. La diferencia, en cuanto a la susceptibilidad de diversos animales, de permitir la multiplicación de parásitos tales como el Toxoplasma gondii, había sido demostrado en células no fagocíticas pero no en células cardíacas (Chinchilla et al. 1995). Aunque conocido el patrón antes mencionado, no se conoce de trabajos en los que se comparan el crecimiento de $T$. cruzi en células de corazón de roedores. Con anterioridad se había informado de la resistencia natural de la rata blanca ante la multiplicación de algunos protozoarios (Chinchilla et al. 1981, 1995) lo cual se correlaciona con los resultados aquí obtenidos, ya que se observó que las células cardiacas de rata son también muy resistentes a la multiplicación de T. cruzi. Probablemente esta resistencia natural está genéticamente determinada y no depende de factores extrínsecos provenientes de la capacidad de infección del parásito. Está generalmente aceptado que las propiedades de la infección de $T$. cruzi son el resultado de la interrelación entre cepas de parásito y las especies de hospederos. Los resultados obtenidos en este estudio parecen ratificar este principio. Según los resultados T. Cruzi (UES) parece ser una cepa con una tasa alta de multiplicación en las células cardiacas de ratón $\mathrm{C}_{3} \mathrm{H}$, además de poseer una mayor capacidad de invadir dicho tipo celular que T. cruzi (TCR-4). Estas diferencias entre la virulencia de estas cepas en cultivos in vitro de células de corazón y debido a la alta capacidad de infectar células cardiacas por parte de la cepa UES, parece conveniente realizar investigaciones de diferenciación de biodemas de cepas aisladas de pacientes salvadoreños con enfermedad de Chagas crónica y analizar mediante un modelo animal, la predilección por el tejido cardíaco.

\section{AGRADECIMIENTOS}

Este estudio fue financiado en parte por la Vicerrectoría de la Universidad de Costa Rica a través del proyecto No. 803-A2-042 y de acuerdo con el convenio Universidad de Costa Rica-DAAD. 


\section{RESUMEN}

Dos cepas de Trypanosoma cruzi, una originaria de Costa Rica (TCR-4) y otra de El Salvador (UES-1) fueron comparadas in vitro en cuanto a su infectividad y multiplicación en células miocárdicas de ratón blanco NGP, ratón $\mathrm{C}_{3} \mathrm{H}$ y rata Sprague Dowley con el fin de determinar la resistencia natural de las células de estos animales. Las células miocárdicas fueron cultivadas en cubreobjetos y posteriormente infectadas con tripomastigotos sanguíneos de la cepa respectiva en una relación de un parásito por célula miocárdica y posteriormente incubados a $37{ }^{\circ} \mathrm{C}$ con $10 \%$ de $\mathrm{CO}_{2}$ en atmósfera húmeda. Para determinar la capacidad infectante y multiplicación intracelular se analizaron muestras a las 24, 72, 96 y $120 \mathrm{~h}$ de infección, determinándose la curva de crecimiento y la tasa de multiplicación intracelular. Se encontró que las dos cepas fueron capaces de multiplicarse en las células miocárdicas de ratón $\mathrm{C}_{3} \mathrm{H}$, muy poco en ratón NGP y prácticamente nada en rata, lo que sugiere una resistencia natural de las células de estos últimos dos animales. La cepa UES-1 tuvo una tasa de multiplicación e invasión mayor $(\mathrm{p}=0.001)$ sugiriendo por lo tanto una virulencia, al menos in vitro superior a la cepa TCR-4.

Palabras clave: Trypanosomatidae, Trypanosoma cruzi, células cardiacas, cultivo de células.

\section{REFERENCIAS}

Andrade, S.G. 1999a. Trypanosoma cruzi: clonal structure of parasite strains and the importance of principal clones. Mem. Inst. Oswaldo Cruz. 94 (Suppl 1.): 185-187.

Andrade, Z.A. 1999b. Immunopathology of Chagas disease. Mem. Inst. Oswaldo Cruz. 94 (Suppl 1.): $71-80$.

Araujo, F.G., D.M. Williams, F.C. Grumet \& J.S. Remington. 1976. Strain dependent differences in murine susceptibility to Toxoplasma. Infect. Immun. 13: $1528-1530$.

Bloch, M. 1982. Conclusiones del estudio de la tripanosomiasis aguda y crónica. Rev. Inst. Invest. Med. 11: 180-183.

Bogdan, C. \& M. Röllinghoff. 1999. How do protozoan parasites survive inside macrophages. Parasitol. Today. 15: 22-28.

Calderón-Arguedas, O., A. Troyo, I. Valerio \& M. Chinchilla. 2002. Heterogeneidad clonal en epimastigotos de una cepa centroamericana de Trypanosoma cruzi (Kinetoplastida: Trypanosomatidae). Parasitol. Latinoam. 57: 40-45.

Chinchilla, M., M. Alfaro \& O.M. Guerrero. 1981. Adaptación natural de la rata blanca a Toxoplasma gondii. Rev. Biol. Trop. 29: 273-282.

Chinchilla, M., L. Reyes \& O.M. Guerrero. 1995. Resistence to intracellular parasites correlates with species differences in ability of macrophages to inhibit parasite replication. Immunol. Infect. Dis. 5: 83-87.

De Luca D’oro, G.M., C.N. Gardenal, B. Berret \& J.V. Crisci. 1993. Genetic structure of Trypanosoma cruzi populations for Argentina estimated from enzyme polymorphism. Parasitol 107: 405-410.

DosReis, G.A. 1997. Cell-mediated immunity in experimental Trypanosoma cruzi infection. Parasitol. Today. 13: $335-342$.

Israelski, D.M., F.G. Araujo, J.S. Wachtel, L. Heinrichs \& J.S. Remington. 1990. Differences in microbicidal activities of human macrophages against Toxoplasma gondii and Trypanosoma cruzi. Infect. Immun. 58: 263-265.

Macedo, A.M. \& S.D.J. Pena. 1998. Genetic variability of Trypanosoma cruzi: implications for the pathogenesis of Chagas disease. Parasitol. Today. 14: 119-123.

Magalhaes, J.B., S.G. Andrade \& I. Sherlock. 1996. Trypanosoma cruzi strains: behavior after pasaje into autochtonous or foreign species of triatomine (Biological and biochemical patterns). Rev. Inst. Med. Trop. Sao Paulo 38: 23-28.

Postani, M., M.R. Arnaiz \& L.E. Fichera. 1999. Respuesta de las células cardiacas a la infección con Trypanosoma cruzi. Medicina. 59 (Suppl II.): 57-62.

Reyes, L. \& M. Chinchilla. 1987. Growth inhibition of Trypanosoma cruzi in cultured murine myocardial cells mediated by a specifically induced lymphokine. Infect. Immun. 55: 1513-1516.

Sadigursky, M. 1999. Evolution on the immunopathology of Chagas disease. Mem. Inst. Oswaldo Cruz. 94:277-278.

Scharfstein, J. \& A. Morrot. 1999. A role for extracellular amastigotes in the immunopathology of Chagas` disease. Mem. Inst. Oswaldo Cruz. 94: 51-63.

Zingales, B. \& W. Colli. 1985. Trypanosoma cruzi: interaction with Host Cells. Cur. Top. Microbiol. Immnol. 117: 129-52. 